\title{
INTERPRETATIVE MODELING OF IMPURITY TRANSPORT AND TUNGSTEN SOURCES IN WEST BOUNDARY PLASMA
}

G. CIRAOLO, S. DI GENOVA, H. YANG, A. GALLO, N. FEDORCZAK, H. BUFFERAND, J.P. GUNN, P. TAMAIN, R. GUIRLET, C. GUILlEMAUT, C. DESGRANGES, C. BOURDELlE, E. TSITRONE, J. BUCALOSSI AND WEST TEAM

CEA, IRFM

Saint-Paul-Lez-Durance, France

Email: guido.ciraolo@cea.fr

S. DI GENOVA, M. SCOTTO D'ABUSCO, E. SERRE

M2P2, AIX-MARSEILLE UNIVERSITE, CNRS, CENTRALE MARSEILLE

Marseille, France

Y. MARANDET, M. RAGHUNATHAN, A. SEPETYS

PIIM, AIX-MARSEILLE UNIVERSITE, CNRS

Marseille, France

J. ROMAZANOV, A. KIRSCHNER, S. BREZINSEK

FZ JULICH GmbH,

Julich, Germany

\begin{abstract}
The contamination of core plasma by high-Z impurities, especially tungsten (W), is the main reason of very high level of radiated power in WEST experiments. Intrinsic light impurities, mainly oxygen and carbon, play a dominant role in the sputtering of $\mathrm{W}$ on plasma facing components. In this contribution, we present a detailed analysis of WEST experiments supported by numerical modeling performed with the transport code SOLEDGE-EIRENE providing a clear picture of light impurities transport and poloidal distribution. Moreover, making use of SOLEDGE-ERO2.0 simulations, possible strategies to reduce core contamination due to $\mathrm{W}$ penetration are presented.
\end{abstract}

\section{INTRODUCTION}

The contamination of core plasma by high-Z impurities, especially tungsten (W), is the main reason of very high level of radiated power in WEST [1] experiments. Determining the main sources of core contamination is indeed a key aspect in preparing a high confinement scenario for the second phase of WEST operation that will start at the end of 2021. Intrinsic light impurities, mainly oxygen and carbon, play a dominant role in the sputtering of $\mathrm{W}$ on plasma facing components (PFCs) and it is crucial to investigate their transport and spatial distribution in edge and scrape-off Layer (SOL) plasmas. In this contribution, we present a detailed analysis of WEST experiments supported by numerical modeling performed with the transport code SOLEDGE-EIRENE. SOLEDGE $[2,3]$ is a unique numerical tool for this kind of studies for two main reasons: firstly, it handles complex and realistic wall geometries thanks to the penalization technique allowing us to properly taking into account the interaction between the plasma and the multiplicity of objects located in the vessel. Secondly, with the recent implementation of multi-fluid collisional closure it is now possible to estimate properly the parallel dynamics and poloidal distribution of light impurities in the edge and SOL plasmas, determined by the competition between thermal gradient and friction forces, without relying on the trace approximation. Both from Vacuum UltraViolet (VUV) diagnostic measurements and simulation results a strong asymmetry between oxygen distribution between the inner and outer targets is found in WEST plasmas. The impact on W sources and transport is investigated using ERO2.0 simulations. In the final part of the contribution a comparison between attached and semidetached plasma is analyzed in terms of W sources and core contamination and a first comparison with experimental results from WEST is shown. 


\section{EXPERIMENTAL INVESTIGATION OF OXYGEN DISTRIBUTION IN WEST DIVERTOR PLASMA AND COMPARISON WITH SOLEDGE SIMULATION RESULTS}

WEST is a MA class superconducting tokamak (plasma current $\mathrm{I}_{\mathrm{p}}=0.5-1 \mathrm{MA}$, toroidal field $\mathrm{B}=3.7 \mathrm{~T}$, major radius $\mathrm{R}=2.5 \mathrm{~m}$, minor radius a $=0.5 \mathrm{~m}$ ), equipped with two up-down symmetric divertors. We focus on a series of discharges related to the experiment on "High power test of ITER-like plasmafacing components, exposure of pre-damaged PFC", supported by the EUROfusion program that took place in 2019 campaign. During these discharges, 4 MW have been injected for few seconds, in lower single null, L-mode plasmas with $2.3 \mathrm{MW}$ of total radiated power, plasma current of $500 \mathrm{kA}$ with a height of the X point of about $115 \mathrm{~mm}$ above the lower divertor target plate. The line integrated density measured by the interfero-polarimeter system [4] is $n_{\text {lid }}^{\text {main }}=4 \times 10^{19} \mathrm{~m}^{-2}$ in the main plasma while in the lower divertor it is $n_{\text {lid }}^{\text {div }}=3-4 \times 10^{18} \mathrm{~m}^{-2}$. In particular, as reference case, we consider the plasma discharge \#54067 which is characterized by stable L-mode, and relevant measurements by VUV spectroscopy system on oxygen distribution in divertor region [5]. The time traces for integrated line density and input power and radiated one are reported in Figure 1.
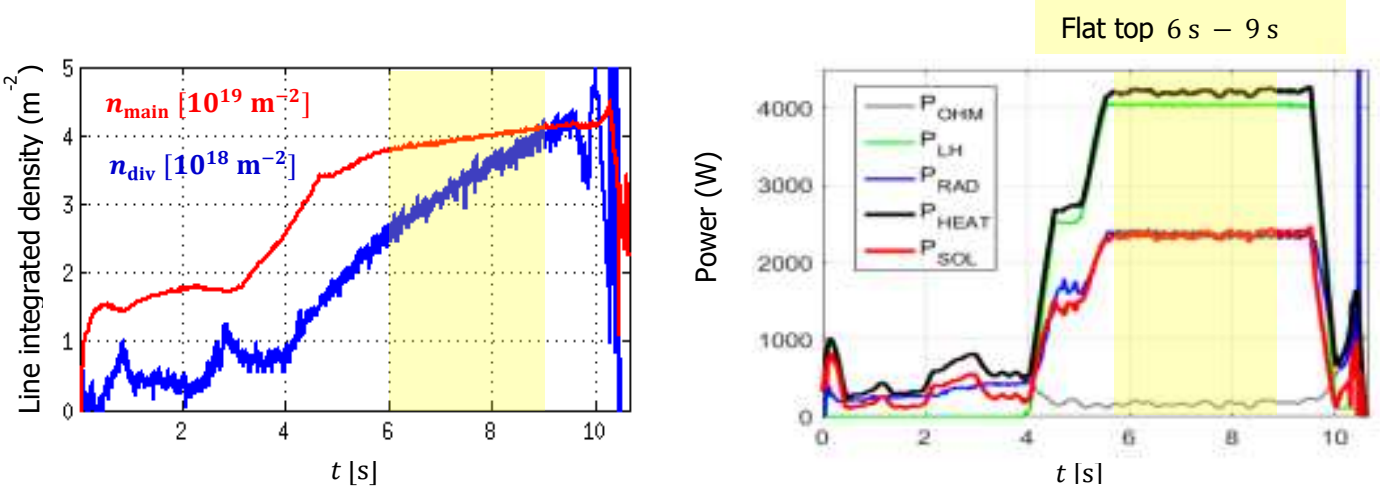

FIG 1: Left panel: time traces of main (red) and lower divertor (blue) integrated line density for the WEST plasma discharge \#54067. Right panel: time traces for the same discharge of total injected power (black) given by the sum of LHCD power (green) and Ohmic one (grey), as well as the total radiated power (blue) and Psol (red), the estimated power flowing into the SOL.

The input parameters for SOLEDGE simulations are the separatrix density at the outer mid-plane and the total injected power into the simulation domain that have been obtained combining measurements from reciprocating Langmuir probe, fast-sweep reflectometry and bolometry diagnostics. More precisely the input power $P_{\text {sol }}$ for SOLEDGE simulation is obtained considering the power balance between the total heating one, $P_{i n j}=P_{L H}+P_{O h m}$, minus the radiated power in the bulk, $P_{\text {rad,bulk, and the }}$ power losses due to magnetic ripple and the generation of fast decoupled electrons, estimated to be approximately equal to $10 \%$ of the injected $P_{L H}$ power [6]. For the discharge under consideration this gives a $P_{\text {sol }}=2.3 \mathrm{MW}$ which in the simulation is equally distributed between main ion and electron channels. Radial transport coefficients for plasma density and temperature have been settled to typical L-mode discharge values, i.e. $\mathrm{D}=1 \mathrm{~m}^{2} / \mathrm{s}$ and $\chi=2 \mathrm{~m}^{2} / \mathrm{s}$ respectively, values from which the SOL width recovered in the simulations is in agreement with the one measured in WEST experiments. In order to determine the percentage of light impurities present in the discharge, we consider simulations with oxygen as an effective medium $\mathrm{Z}$ charge impurity for sputtering. A parametric scan on oxygen concentration is performed, assuming from $1 \%$ to $4 \%$ of oxygen injected in SOLEDGE at the inner boundary of simulation domain. The simulations with $2 \%$ of oxygen are the ones matching quite well both divertor target profiles as well as total radiated power, as reported on figures 2 and 3 . More precisely, in Fig. 2 are presented the 2D poloidal maps of main ion density and of the total radiated power, as well as an image obtained from visible camera during the flat top of the discharge. In addition to the striking common feature of the simulation and the experiment of a visible radiating layer in the edge region of the high field side, a more quantitative comparison has been performed on the total radiated power, equal to $780 \mathrm{~kW}$ in SOLEDGE simulation domain which is in quantitative agreement 
with the experimental one obtained using bolometry measurements related to the upper and lower divertor regions.
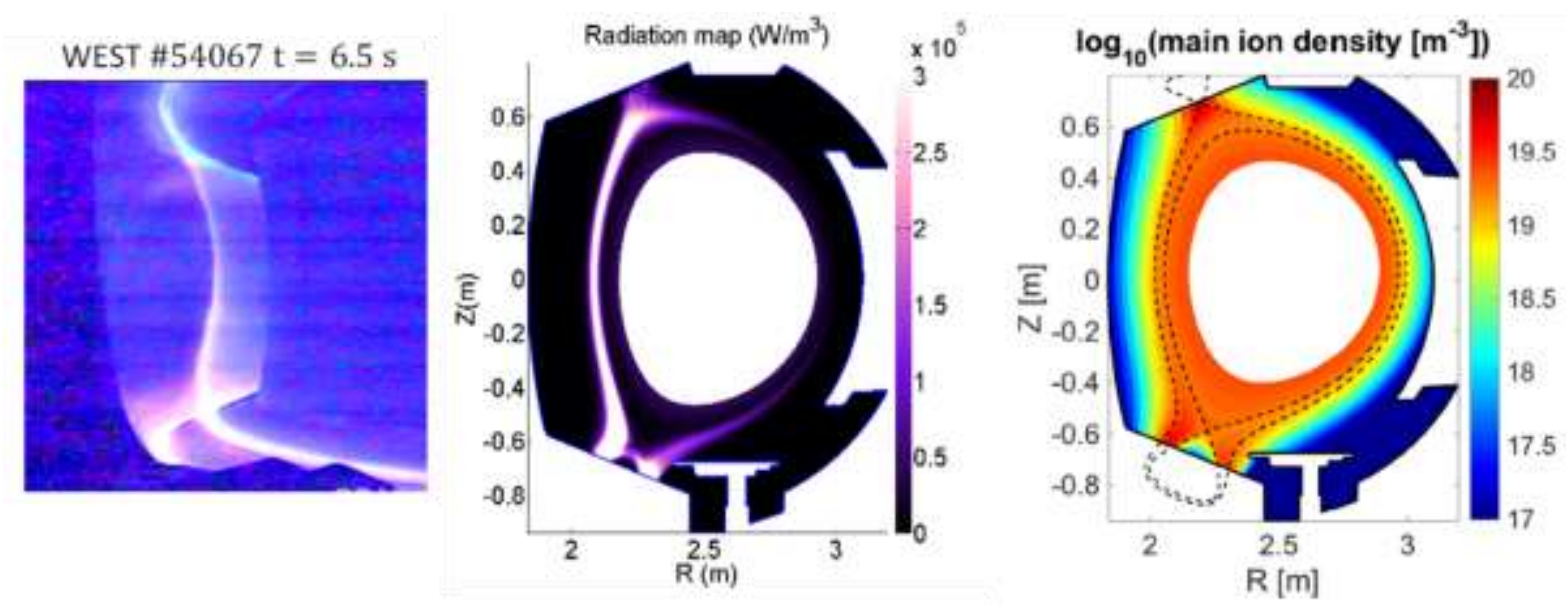

FIGURE 2: Left panel: Visible camera image obtained during the flat top phase of the discharge \#54067 showing strongly radiating layers both along the lower divertor targets as well as in the edge region of the high field side. Central panel: Poloidal map of the radiating power obtained from the SOLEDGE simulation for the flat top phase of the discharge \#54067, reproducing qualitatively the radiating layers observed in the experiment. The total amount of radiated power in the computational domain, $P_{\text {rad }} S O L$, is equal to $780 \mathrm{~kW}$ and it is in good agreement with the radiated power measured in the experiment for the edge and SOL regions. Right panel: poloidal map of main ion density obtained in the SOLEDGE simulation.

The simulations results are then analyzed (see Fig.3) comparing electron density and temperature profiles at the lower divertor targets with experimental data from locally embedded Langmuir probes as well as the perpendicular heat flux with respect to the one obtained from thermocouples.
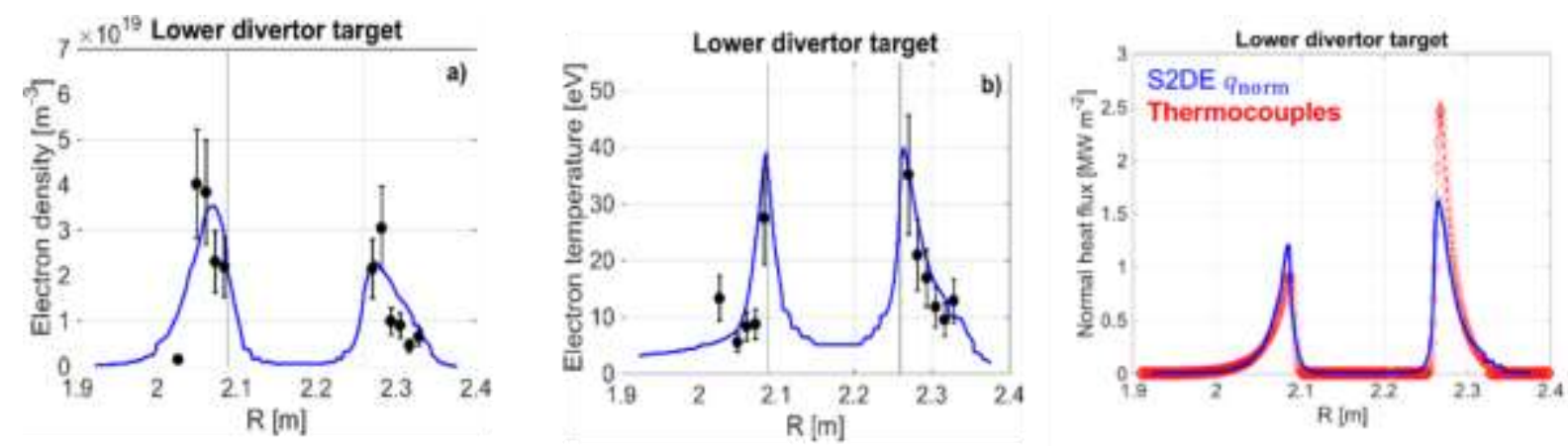

FIGURE 3: Left and central panels: Profiles of respectively electron density and electron temperature at the lower divertor for WEST discharge \#54067. In blue solid line SOLEDGE simulation, in black symbols the LP measurements. Right panel: heat flux (component normal to the surface) along the lower divertor from SOLEDGE simulation (blue solid line) and from thermocouples (red circle symbols).

The agreement is very good for electron temperature profiles and also for electron density profiles while for heat flux the SOLEDGE simulations reproduces the inner/out asymmetry but with a smaller pick value for the heat flux on the outer target.

To go further into the simulation/experiment comparison we look at the poloidal distribution of oxygen. The simulation results show a strong asymmetry between oxygen concentrations at the inner divertor target with respect to the outer one. This asymmetry has been measured in the experiments thanks to the WEST VUV spectroscopy system with a moving line of sight in the poloidal plane, allowing one to retrieve the relative information on the angular position of the oxygen light emission (see Fig. 4). 

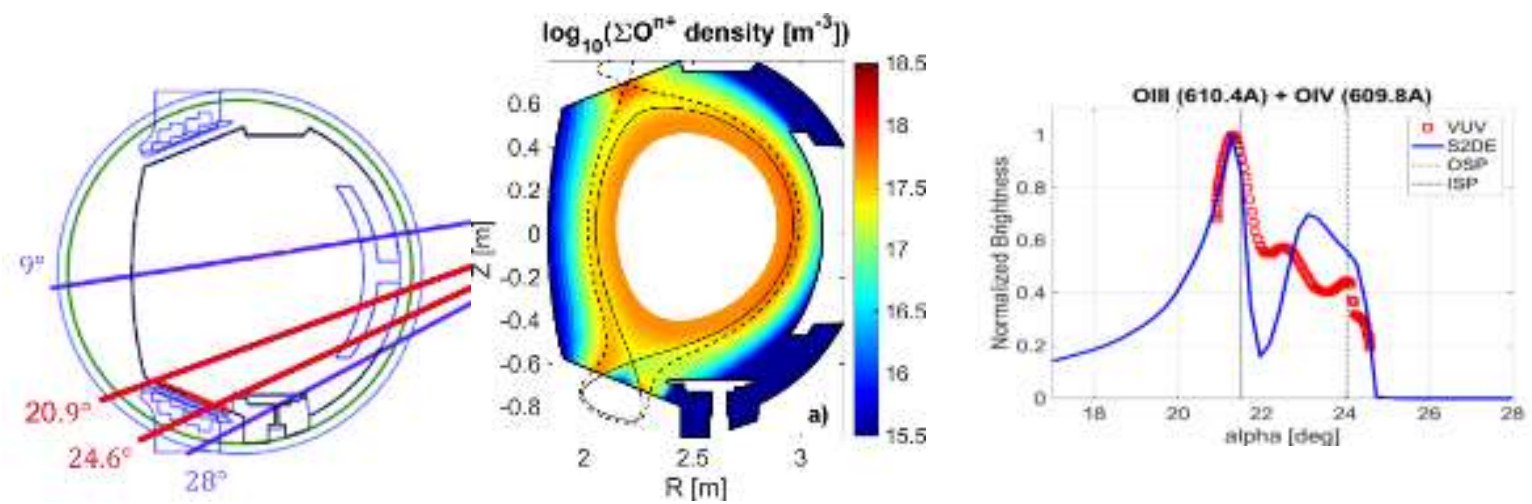

Figure 4: Left panel: VUV system in scanning mode. Central panel: $2 D$ poloidal map of oxygen density from SOLEDGE2D simulation. Right panel: Comparison of "poloidal" profiles of oxygen in lower divertor (not absolutely calibrated). Experimental data from VUV system are reported with red symbols while the solid blue line represents results from SOLEDGE simulation.

The detailed force balance analysis on simulation results shows that thermal gradient forces are stronger at the outer divertor target pushing the oxygen toward upstream location and producing its depletion in this region. This result is in agreement with $\mathrm{O} I V\left(\mathrm{O}^{3+}\right)$ line from VUV spectroscopy signals, as showed in Fig. 4.

The influence of this light impurity on tungsten sputtering and consequent transport into the plasma was investigated post-processing SOLEDGE simulations through the Monte Carlo wall impurities tracker ERO2.0 [7, 8]. Sputtering yield coefficients were taken from TRIM simulations results, sputtering distributions for energy and azimuthal angle were taken to be equal to the standard Thompson and cosine distributions respectively, the radial diffusion coefficient $\mathrm{D}_{\mathrm{w}}$ for $\mathrm{W}$ transport was set to be equal to $1 \mathrm{~m}^{2} / \mathrm{s}$ coherently with the one used in the SOLEDGE simulations for this plasma background. We report on Fig. 5 a poloidal map of $\mathrm{W}$ density using SOLEDGE plasma background with a $2 \%$ of Oxygen. Indeed in the version of ERO2.0 we used we cannot specify the poloidal distribution of the impurities obtained in SOLEDGE simulations along the wall but we have to set a fixed one that we have chosen as the have a first global picture of $\mathrm{W}$ transport and its penetration into the core region.

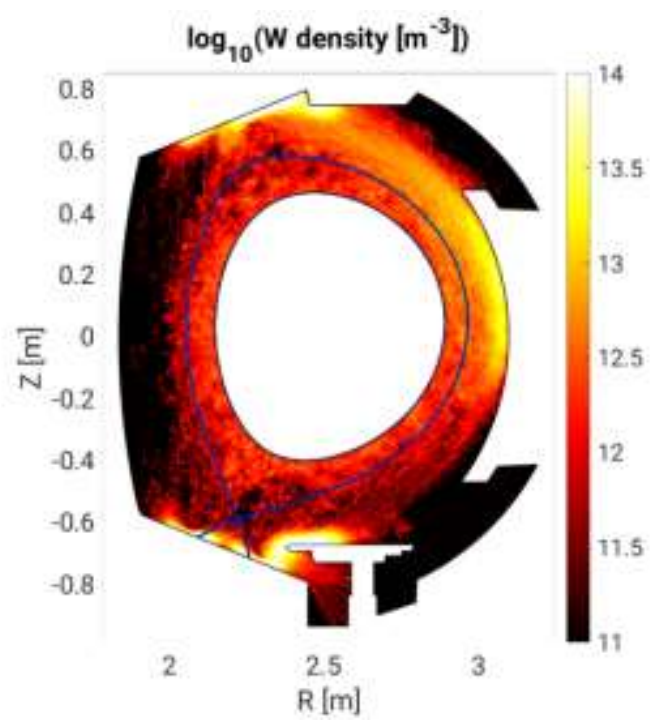

Figure 5: Poloidal map of $W$ density computed with ERO2.0 using the plasma background provided by SOLEDGE for the shot \#54067and presented in Figs 2. The percentage of oxygen has been set equal to $2 \%$ as an input for the ERO2.0 simulations.

One can observe strong $\mathrm{W}$ sources predicted by ERO2.0 at the bottom and up divertor as well as on the baffle and at the antenna limiter locations. Concerning $\mathrm{W}$ transport, the screening seems quite efficient 
at the bottom divertor as well as on the baffle while upper divertor and antenna limiter seem to be much less screened, causing potentially the strong $\mathrm{W}$ core contamination. To go further into the analysis of the main PFC contributors for $\mathrm{W}$ core contamination we performed a series of simulations activating one main PFC at time and using for each of them the estimated oxygen distribution in that region from the plasma background (composed from deuterium and oxygen) computed with SOLEDGE where the percentage of oxygen founded in these main regions was varied between $1 \%$ on the outer target of the low divertor up to $2.4 \%$ on the upper divertor region. The results on the $2 \mathrm{D}$ poloidal $\mathrm{W}$ density are shown on Fig. 7
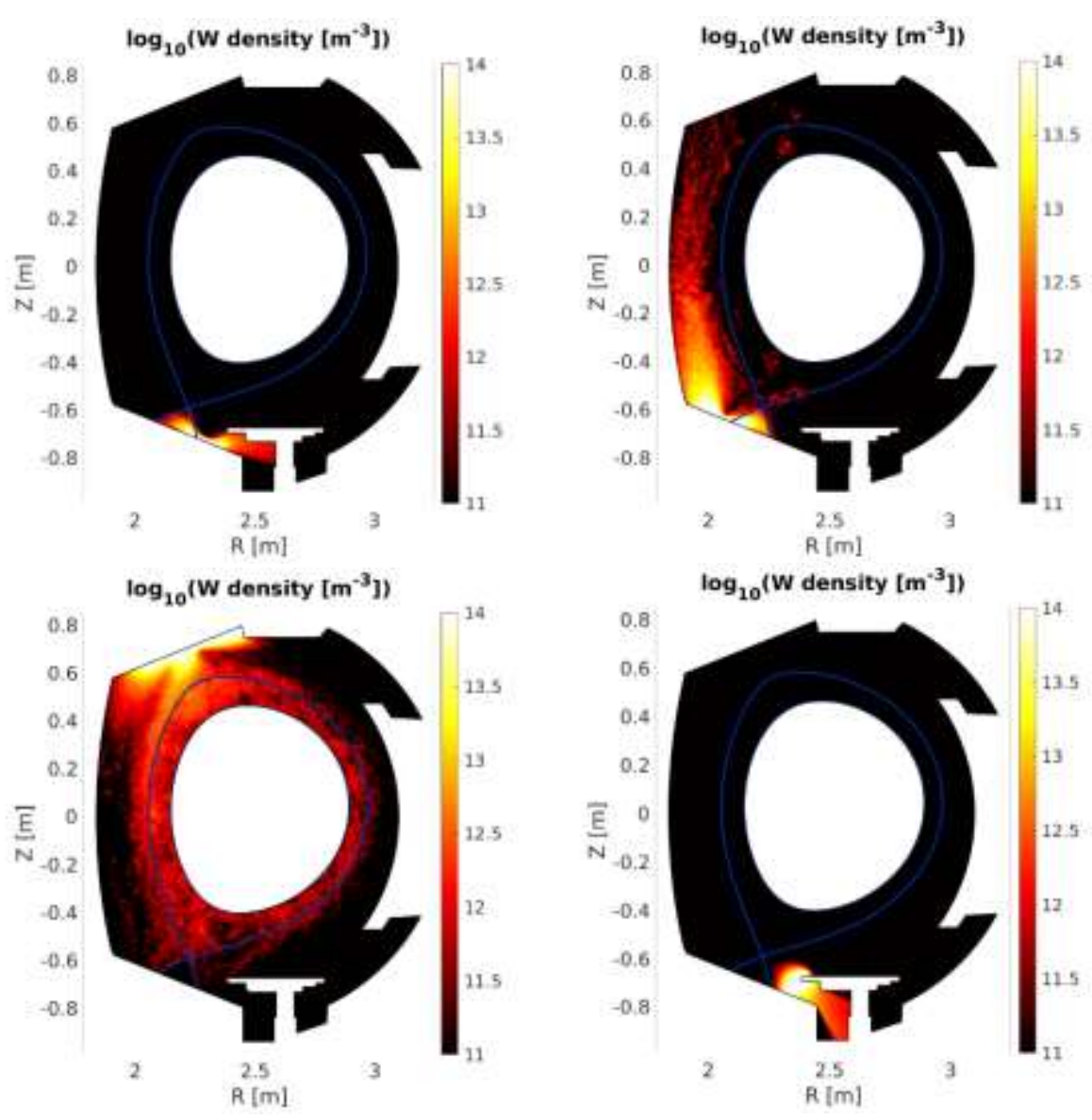

Figure 6: $2 D$ plot of $W$ density obtained with ERO2.0 activating one main PFC region and considering the SOLEDGE background for the WEST shot \# 54067. Top left (right) panel: W poloidal distribution obtained considering only the outer (inner) target region of the lower divertor and using $1 \%(1.8 \%$ respectively) of oxygen in the ERO2.0 simulation. Bottom left panel: W poloidal distribution obtained considering only the upper divertor region and using 2.4\% of oxygen in the ERO2.0 simulation. Bottom right panel: $W$ poloidal distribution obtained considering only the baffle region and using $2.1 \%$ of oxygen in the ERO2.0 simulation

As a first analysis on the $\mathrm{W}$ sources, starting from the poloidal maps of $\mathrm{W}$ obtained with ERO2.0 we have applied synthetic diagnostics to reproduce the visible spectroscopy measurements like in WEST VS system. The synthetic diagnostics procedure consists in post-processing the $2 \mathrm{D}$ emission map of a certain visible wavelength of W calculated by ERO2.0 using SYNDI (a Monte Carlo beam tracer) [9], in order to obtain the line-integrated flux of photons on the WEST visible spectroscopy system lines of sight. The wavelength for which the visible spectroscopy (VS) was analyzed is the standard WI line $400.9 \mathrm{~nm}$. In particular we have computed the photon fluxes of WI associated to the regions of the lower divertor (inner strike point and outer strike point as separate regions) and upper divertor, see Fig. 7. 


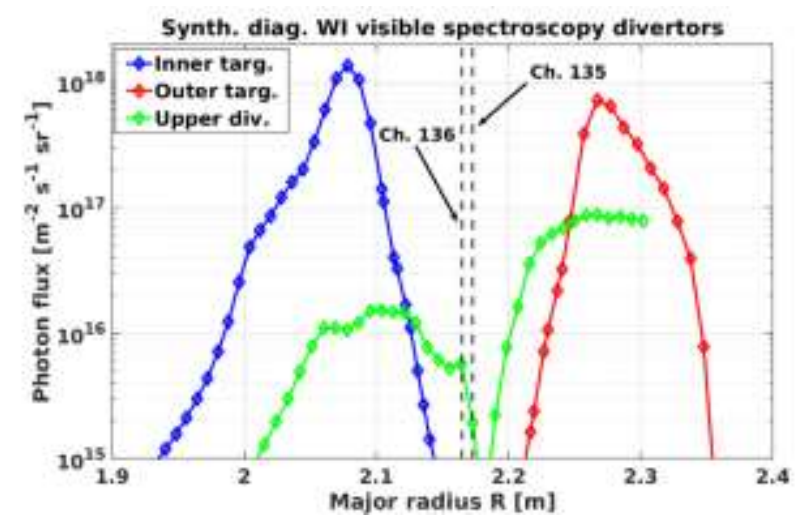

Figure 7: Line-integrated photon fluxes for the WI line $400.9 \mathrm{~nm}$ obtained applying synthetic diagnostic tool SYNDY on the ERO2.0 results for divertor regions (in blue the bottom inner target region, in red the bottom outer target one and in green the upper divertor region).

On the lower divertor the peak value on the inner strike point region is slightly higher than on the outer one. At the same time the ratio between the peak values on the lower divertor and higher divertor varies from one to two orders of magnitude which seems in line with a first global analysis of WEST database for that campaign (we remind here that unfortunately for this particular shot the signals of visible spectroscopy are not available). More precisely the channels that are usually considered for the experimental analysis of upper divertor are Ch. 136 and 135, indicated with black dashed lines on Fig. 7. One can remark that using these lines the ratio between upper and lower divertor is in the range of 100 but that for other regions it is reduced to 10 . It will be interesting for further studies to investigate the ensemble of upper divertor channels in WEST experiments to verify if the sources on the upper divertor can be so high.

We focus now on the transport of $\mathrm{W}$ indicated from ERO2.0 simulations. As we can see in Fig. 6, even if the $\mathrm{W}$ source on the outer target of the bottom divertor as well as of the baffle are quite important, these elements are screened very well from the plasma and the $\mathrm{W}$ do not penetrate into the core region. On the contrary it seems quite important the $\mathrm{W}$ core contamination due to the inner target of the bottom divertor as well as from the upper divertor region. This can be due both to the impact of drifts on W transport (considered in ERO2.0 but not in SOLEDGE plasma background), as for example the downward curvature drift, but also to the wall geometry, with the presence of a baffle in the lower divertor region and not in the upper one. A detailed analysis of the impact of these several players in the $\mathrm{W}$ transport and core contamination is ongoing and will presented in a future work.

We would like to point out that here we do not consider the same analysis for the antenna limiter due to the strong uncertainties induced from the fact that the plasma background is obtained using SOLEDGE in the 2D axisymmetric approximation, while in the reality the antenna limiter is localized toroidally with a very small angular extension. The consequence is the overestimation of its contribution to $\mathrm{W}$ sputtering and core contamination.

In the following section we will investigate numerically a possible scenario to reduce the $\mathrm{W}$ sources and consequently the contamination of the core plasma.

\section{NUMERICAL INVESTIGATION OF THE IMPACT OF SEMIDETACHED PLASMA ON W SOURCES, TRANSPORT AND CORE CONTAMINATION}

The development of a robust semi-detached divertor plasma scenario should permit to operate below the sputtering threshold of light impurities, reducing strongly $\mathrm{W}$ sources and opening the way to high confinement scenarios. In this respect we have performed a first series of SOLEDGE-ERO2.0 simulations in order to have a first estimation of the differences between these two scenarios on W sources and transport as well as core contamination. First we have computed with SOLEDGE two plasma backgrounds for WEST, one attached with a temperature at the strike points of the lower divertor equals to about $10 \mathrm{eV}$ and one semi-detached, with a temperature at the inner strike point lower than 2 $\mathrm{eV}$ while on the outer strike point a temperature around $4 \mathrm{eV}$. In both cases the main ion was deuterium 
and $3 \%$ of oxygen was considered. The influence of this light impurity on tungsten sputtering and contamination was investigated post-processing SOLEDGE simulations with ERO2.0. Sputtering yield coefficients were taken from TRIM simulations results, sputtering distributions for energy and azimuthal angle were taken to be equal to the standard Thompson and cosine distributions respectively, $\mathrm{W}$ anomalous diffusion coefficient $\mathrm{D}$ was set to be, coherently with the one used in these SOLEDGE simulations, equal to $0.3 \mathrm{~m}^{2} / \mathrm{s}$. We report on Fig.5 a poloidal map of $\mathrm{W}$ density in the two plasma configurations, attached on the left panel and semidetached on the right one.
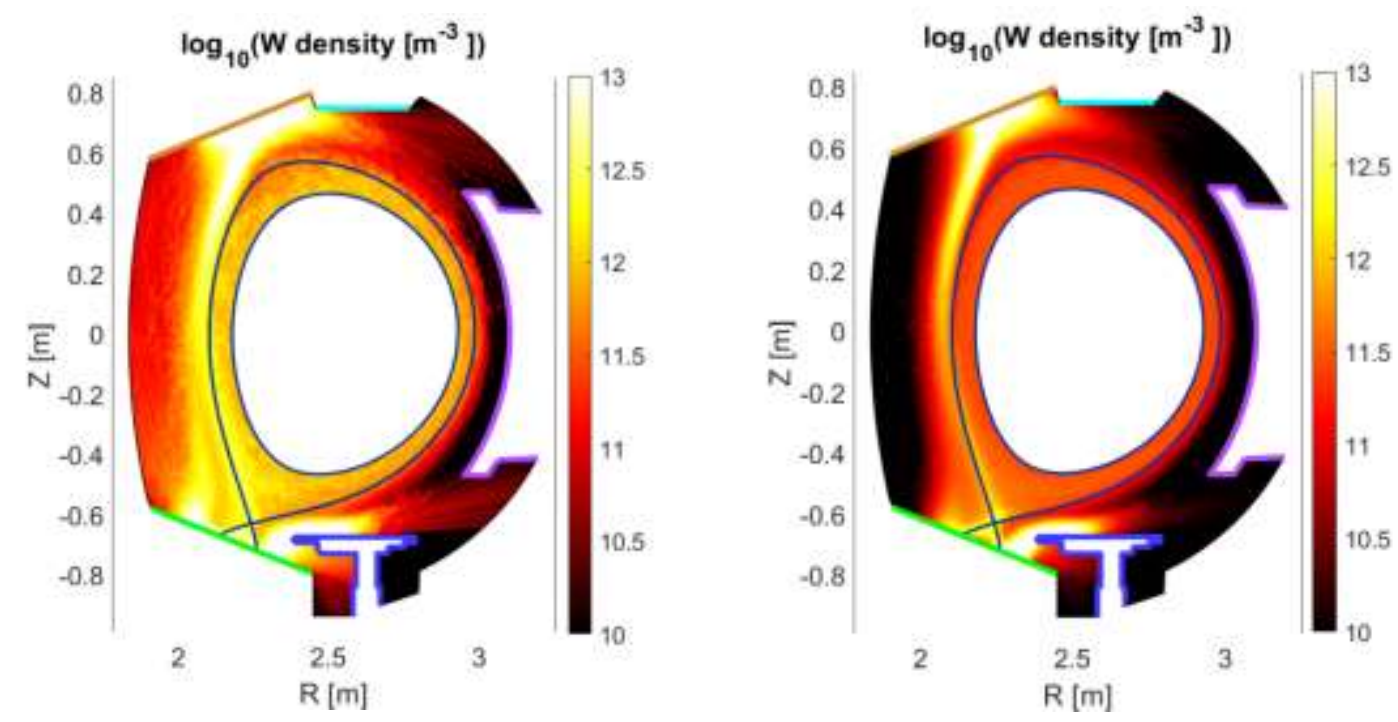

Figure 8: Left panel: Poloidal map of $W$ density computed with ERO2.0 using an attached plasma background provided by SOLEDGE. Right panel: Poloidal map of $W$ density computed with ERO2.0 using a semi-detached plasma background provided by SOLEDGE.

From the 2D map it is clear that for the semidetached plasma background the $\mathrm{W}$ source at the inner target is strongly reduced. To investigate the role of the different regions and main PFC of the tokamak chamber, ERO2.0 simulations were repeated using only one WEST PFC "main region" at a time, and their impact on core contamination is presented in table 1 .

\begin{tabular}{|l|c|c|}
\hline PFC & $\begin{array}{c}\text { Number of } W_{\text {core }} \text { [particles] } \\
\text { for the attached plasma }\end{array}$ & $\begin{array}{c}\text { Number of } W_{\text {core }} \text { [particles] } \\
\text { For the semi-detached plasma }\end{array}$ \\
\hline Inner Target & $1.2 \times 10^{11}$ & $2.7 \times 10^{8}$ \\
\hline Outer Target & $7.5 \times 10^{6}$ & $1.1 \times 10^{8}$ \\
\hline Baffle & negligible & negligible \\
\hline Antenna & $1.1 \times 10^{9}$ & $2.3 \times 10^{7}$ \\
\hline Ceiling & $1.8 \times 10^{9}$ & $7 \times 10^{7}$ \\
\hline Upper divertor & $6.4 \times 10^{12}$ & $2.2 \times 10^{12}$ \\
\hline
\end{tabular}

Table 1: Number of $W$ particles entering the core region coming from different PFC computed using ERO2.0 and related to an attached and semi-detached plasma background.

The core contamination due to $\mathrm{W}$ is computed as the integrated $\mathrm{W}$ density inside the separatrix obtained for each simulation where only one PFC at time was "active", i.e. considered as possible source of W. One notices that for the semi-detached plasma the strong reduction of contamination due to the inner strike point while for the outer one the opposite is true, even if globally the reduction in the total contamination is equal to a factor 2 . We remark also that due to the small value of the radial diffusion coefficient $\mathrm{D}=0.3 \mathrm{~m}^{2} / \mathrm{s}$, the contamination due to the baffle estimated from the simulation in negligible. 
However, using the same plasma backgrounds and increasing the diffusion coefficient to $D=1 \mathrm{~m}^{2} / \mathrm{s}$ the contamination due to the baffle becomes also important, underlying the importance of such parameter in the ERO2.0 simulations.

These predictions have been compared with first experimental evidences about the strong reduction of W sources when the plasma enters in detached phase. In particular we have analysed the WEST shot \#56420 which is characterized for a quite long detached phase with a strong radiation pattern localized around the X-point.

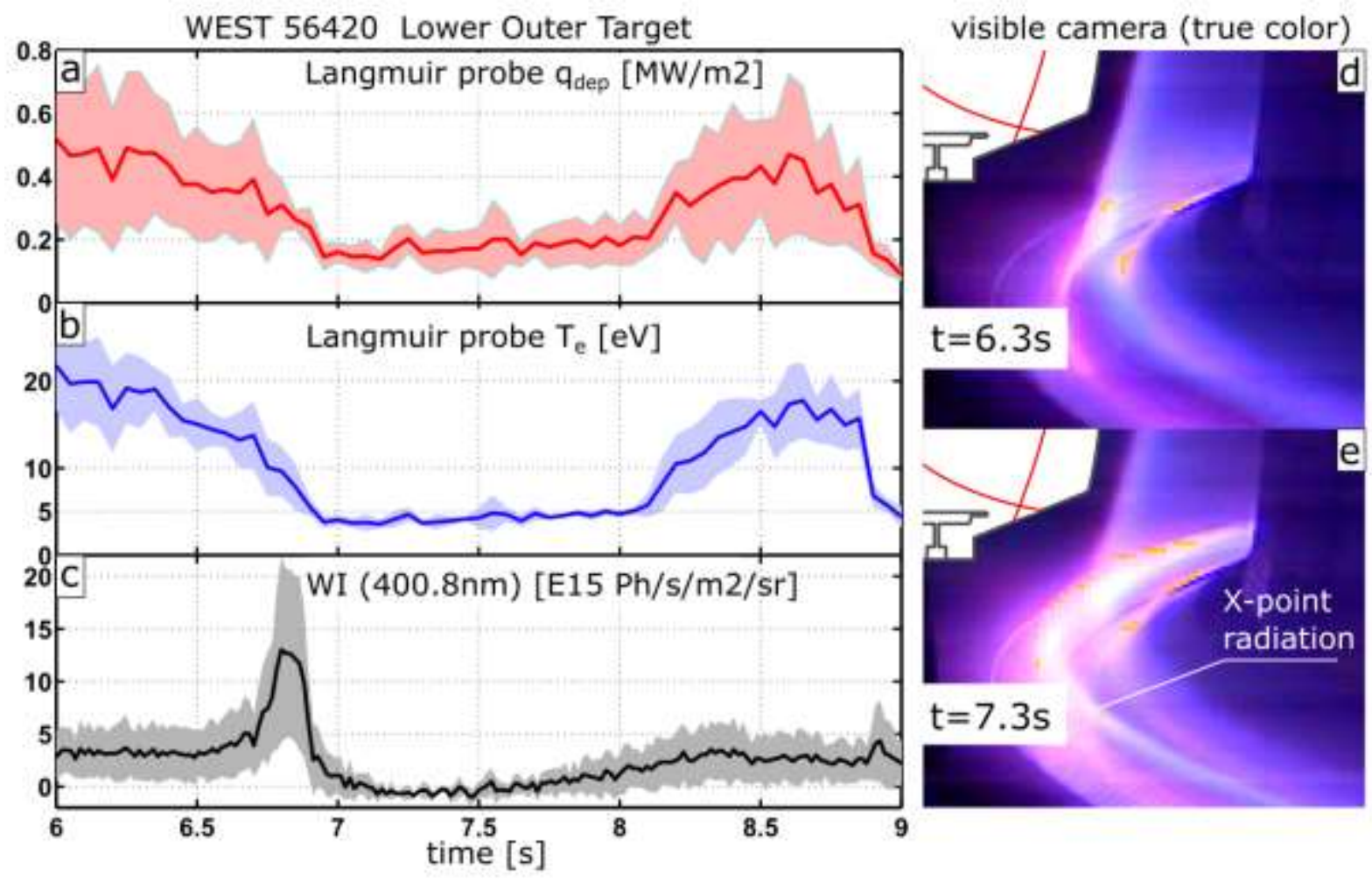

Fig. 9: WEST shot \#56420. Time traces of deposited heat load (a, Langmuir probe), electron temperature (b, Langmuir probe) and neutral tungsten radiance (c, visible spectroscopy) spatially averaged $5 \mathrm{~cm}$ around the outer strike point (colored areas surrounding average curves show the standard deviation in this $5 \mathrm{~cm}$ band around strike point). Visible camera images of the divertor region are shown for $t=6.2 \mathrm{~s}(d)$ and $t=7.3 \mathrm{~s}(\mathrm{e})$, the later exhibiting a localised emission ring possibly close to the X-point. The pulse features a puff ramp in ohmic, with puff injection stopped at 7.5s.

Starting around 6.5s, the outer divertor enters the high recycling regime, with a deposited heat flux and electron temperature dropping rapidly. At the same time, the tungsten source (proxy from line radiance) increases rapidly (consequence of enhanced particle flux not show here), reaching a maximum at $6.8 \mathrm{~s}$ and quickly dropping to zero. This source dynamics is consistent with observations from other devices as AUG, JET and EAST (see for example refs. [10, 11, 12, 13, 14, 15]), and relates to the low electron temperature (below $5 \mathrm{eV}$ ) reached in the very high recycling regime, below the sputtering threshold of light impurities on tungsten. From $\mathrm{t}=6.9 \mathrm{~s}$ to about $8 \mathrm{~s}$, the outer divertor stays in a stable semi-detached regime characterised by a tungsten source that is cancelled, a target electron temperature below $5 \mathrm{eV}$, and a finite heat load $\left(0.2 \mathrm{MW} / \mathrm{m}^{2}\right)$ that is 2 to 3 times lower than before detachment. Additionally, visible camera image of the divertor region exhibits a localised emissive cloud sitting close to the $\mathrm{X}$ point location, away from the targets. This regime may show some characteristics close to the "X-point radiation regime" focused on AUG (see ref. [16]), although it is apparently not a deep detachment regime for this specific WEST experiment. After 8s, the divertor slowly re-attach as a consequence of no sustained gas puffing from $7.5 \mathrm{~s}$. 
These first studies indicate that semidetached steady state plasmas are a possible solution for the reduction of $\mathrm{W}$ core contamination and large radiated power in WEST experiments. Further experimental analysis, using for example visible spectroscopy data, as well as numerical modelling, are ongoing and will help in preparing the phase 2 of WEST operation.

\section{ACKNOWLEDGEMENTS}

This work was granted access to the HPC resources of CINES, under the allocation 2018-A0040510482 made by GENCI and to the HPC resources of Aix-Marseille University financed by the project Equip@Meso (ANR-10EQPX-29-01). This work has been carried out within the framework of the EUROfusion Consortium and has received funding from the Euratom research and training programme 2014-2018 and 2019-2020 under grant agreement No 633053. The views and opinions expressed herein do not necessarily reflect those of the European Commission

\section{REFERENCES}

[1] BUCALOSSI, J. and the WEST team, (2014), Fusion Eng. Design 89, 907-912.

[2] BUFFERAND, H. et al., Numerical modelling for divertor design of the WEST device with a focus on plasma-wall interactions, Nucl. Fus. 55 (2015) 053025

[3] CIRAOLO, G. et al. First modeling of strongly radiating WEST plasmas with SOLEDGE-EIRENE, Nucl. Mat. \& En., 20, (2019) 100685

[4] GIL, C. et al, Renewal of the interfero-polarimeterdiagnostic for WEST, Fusion Eng. Des. 140 (2019) 81-91

[5] GALLO, A. et al, Interpretative transport modeling of the WEST boundary plasma: main plasma and light impurities, Nucl. Fusion 60 (2020) 126048

[6] BASIUK, V. et al, Studies of suprathermal electron loss in the magnetic ripple of tore supra, Nucl. Fus. 41 (2001), 477

[7] ROMAZANOV, J. et al, First ERO2.0 modeling of Be erosion and nonlocal transport in JET ITER-like wall, Phys. Scr. (2017) 2017014018

[8] SEPETYS, A., Tungsten sources in the divertor and the main chamber and contamination of the WEST tokamak plasmas, PhD manuscript (2019), available at https://hal.archives-ouvertes.fr/tel-02612763

[9] FEDORCZAK, F. and MAO, R., Private communication (2019)

[10] REIMOLD, F. et al, Divertor studies in nitrogen induced completely detached H-modes in full tungsten ASDEX Upgrade, Nucl. Fusion 55, (2015) 033004

[11] KALLENBACH, A. et al, Partial detachment of high power discharges in ASDEX Upgrade, Nucl. Fusion 55 (2015) 053026

[12] NEU, R. et al, Overview on plasma operation with a full tungsten wall in ASDEX Upgrade, J. Nucl. Mat. 438 (2013) S34

[13] MATTHEWS, G. F., et al, The second phase of JET operation with the ITERlike wall, Phys. Scr. T159 (2014) 014015

[14] BREZINSEK, S. et al, Erosion, screening, and migration of tungsten in the JET divertor, Nucl. Fusion 59 (2019) 096035

[15] WANG, L. et al, Advances in plasma-wall interaction control for Hmode operation over $100 \mathrm{~s}$ with ITER-like tungsten divertor on EAST, Nucl. Fusion 59 (2019) 086036

[16] BERNERT, M. et. Al Control of the X-point radiator in fully-detached ASDEX Upgrade H-mode plasmas. Preprint: 2020 IAEA Fusion Energy Conference (2021) EX/7-3 